\title{
İRSETCONF
}

\section{EFFECT OF THE POLARIZATION OF TRIPOLAR ELECTRODES ON ELECTRICAL NERVE BLOCK IN-VIVO}

\author{
Büşra Şahin ${ }^{1 *}$, F. M. Betül Erol ${ }^{*}$, Zafer Soybaş ${ }^{1}$, Sefa Şimşek ${ }^{1}$, Rohat Melik ${ }^{1+}$ \\ ${ }^{1}$ TOBB University of Economics \& Technology, Ankara, Turkey \\ Department of Electrical and Electronics Engineering, \\ ${ }^{2}$ Hacettepe University, Ankara, Turkey / Faculty of Medicine \\ *Equal Contribution \\ ${ }^{+}$Corresponding Author: Rohat Melik
}

\begin{abstract}
Electrical nerve block is an emerging technique that has many potential advantages and promise novel treatment opportunities. Applying DC-current is one approach to produce nerve block through closing inactivation gates of sodium-channels. However, DC-current can damage the nerve, therefore it is crucial to use the lowest current possible. For this purpose, we used tripolar electrodes to obtain larger surface area and investigated the most effective combination of polarization. We observed that -++, --+, -+-, +-+, +--, ++- polarizations, from proximal-side to distal-side of the sciatic nerve of the frog, exhibit efficient results respectively. In -++ case, as current goes from positive-to-negative pole, the blocking current opposes the action potential current, which promotes blocking. In ++- state; however, the direction of blocking current is same with the action potential current, which makes it harder to achieve full blocking. In conclusion, ++ polarization is the most effective tripolar configuration for electrical nerve block with DCcurrent.
\end{abstract}

Keywords: action potential; blocking of the nerve; direct current; electrode polarity; sciatic nerve 